\title{
On-line maximum-order induced hereditary subgraph problems (Preliminary version)
}

\author{
Marc Demange \\ CERMSEM \\ Universit Paris I* \\ demange@univ-paris1.fr
}

\author{
Xavier Paradon Vangelis Paschos ${ }^{\dagger}$ \\ LAMSADE \\ Université Paris-Dauphine ${ }^{\ddagger}$ \\ \{paradon, paschos\}@lamsade.dauphine.fr
}

\section{Contents}

$\begin{array}{ll}\text { Abstract } & 2\end{array}$

1 Introduction 2

2 On-line maximum-order hereditary subgraph 3

3 On-line independent set $\quad 4$

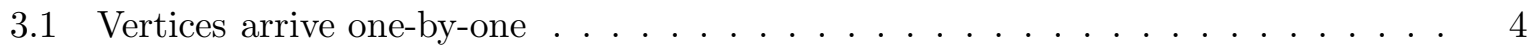

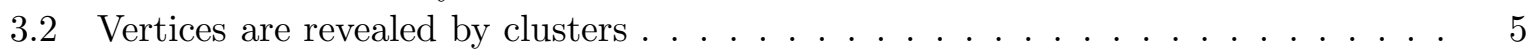

4 On-line maximum clique $\quad 6$

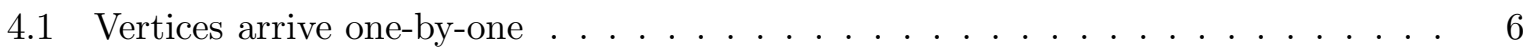

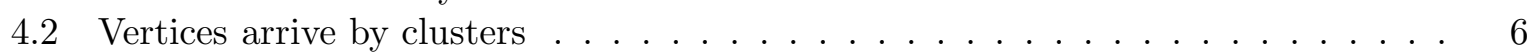

4.2.1 Reducing on-line clique to (off-line) clique . . . . . . . . . . . 6

4.2.2 Reducing on-line independent set to on-line clique . . . . . . . . . 7

${ }^{*}$ Maison des Sciences Economiques, 106-112 boulevard de l'Hôpital, 75647 Paris Cedex 13, France

${ }^{\dagger}$ Author for correspondence

${ }^{\ddagger}$ Place du Maréchal De Lattre de Tassigny, 75775 Paris Cedex 16, France 


\begin{abstract}
We first study the competitivity ratio for the on-line version of the problem of finding a maximum-order induced subgraph satisfying some hereditary property, under the hypothesis that the input graph is revealed by clusters. Then, we focus ourselves on two of the most known instantiations of this problem, the maximum independent set and the maximum clique.
\end{abstract}

\title{
1 Introduction
}

On-line algorithmic is a very active computer science area where one supposes that the instance of a problem is revealed step-by-step and one has, at the end of each step, to irrevocably decide on the part of the final solution dealing with this step.

In this paper, we study an on-line version of the following problem, denoted by HG: given an input-graph $G$, find a maximum-order subgraph of $G$ verifying a non-trivial hereditary property $\pi$. Let $\mathcal{G}$ be the class of all graphs. A graph-property $\pi$ is a mapping from $\mathcal{G}$ to $\{0,1\}$ (for $G \in \mathcal{G}, \pi(G)=1$ iff $G$ satisfies $\pi$ and $\pi(G)=0$, otherwise). Property $\pi$ is hereditary if whenever it is satisfied by a graph it is also satisfied by every one of its induced subgraphs; it is non-trivial if it is true for infinitely many graphs and false for infinitely many ones. The property " $\pi$ : is a clique", or " $\pi$ : is an independent set" are typical examples of such non-trivial hereditary properties. The maximum clique problem, or the maximum independent set problem are typical examples of hereditary induced subgraph problems.

The on-line version of HG, denoted by LHG is defined as the pair (HG, $\boldsymbol{R})$ where $\boldsymbol{R}$ is a set of rules dealing with:

1. information on the value of some parameters of the final graph;

2. how the final graph is revealed.

An on-line algorithm decides at each step which of the vertices revealed during this step will belong to the final solution. The performance of an on-line algorithm $\mathbf{A}$ is measured in terms of the so-called competitivity ratio $c$ defined, for an instance $G$ and a set $\boldsymbol{R}$, as the ratio of the worst ${ }^{1}$ value of the solution computed by $\mathbf{A}$ on $G$ to $\beta(G)$, where $\beta(G)$ is the value of the solution computed by an optimal off-line algorithm on $G$.

In this paper, we consider that the input-graph $G$ is revealed per non-empty clusters, i.e., per induced subgraphs of $G$ together with the edges linking vertices of the last revealed cluster to the already revealed ones. In what follows, we denote by $t(n)$ the number of clusters needed so that the whole graph is completely revealed.

Two well-known particular cases of $\mathrm{HG}$ are the maximum independent set problem (IS) and the maximum clique problem $(\mathrm{Cl})$. The former is the problem of finding a maximum-size subset $V^{\prime} \subseteq V$ of a graph $G(V, E)$ such that $\forall(v, u) \in V^{\prime} \times V^{\prime}, v u \notin E$, while the latter is the problem of finding a maximum-size subset $V^{\prime} \subseteq V$ of a graph $G(V, E)$ such that $\forall(v, u) \in V^{\prime} \times V^{\prime}$, $v u \in E$.

In what follows, we first study LHG. Next we deal with on-line versions of IS and $\mathrm{Cl}$, denoted by LIS and LCl, respectively, under the restrictive case where $t(n)=n$, i.e., when the graph is revealed vertex-by-vertex, and the general one where $t(n)<n$. Finally we study some competitivity preserving reductions for LIS and LCl.

\footnotetext{
${ }^{1}$ Over all the ways $G$ is revealed according to $\boldsymbol{R}$.
} 


\section{On-line maximum-order hereditary subgraph}

We consider that $G$ is revealed by $t(n)$ non-empty clusters. In what follows, we suppose that when we deal with approximation, or competitivity ratios, functions of a certain number of parameters of the final graph, the values of these parameters are known from the beginning of the on-line process. In this paper we consider ratios, functions of $n$ and/or $\Delta(G)$, the maximum degree of the final graph.

Theorem 1. Suppose that $H G$ can be approximately solved by a polynomial time approximation algorithm $\boldsymbol{F A}$ achieving approximation ratio ${ }^{2} \rho(n, \Delta(G)$ ) (decreasing in $n$ and $\Delta(G)$ for a maximization problem). Then, there exists an on-line algorithm $\boldsymbol{L} \boldsymbol{A}$ for $L H G$ achieving, for every instance $G$,

$$
c_{\mathbf{L A}}(G) \geqslant \sqrt{\frac{\rho(n, \Delta(G))}{n t(n)}} .
$$

Proof. (Sketch.) Consider the following algorithm where $r(n)$ is a threshold-function specified later.

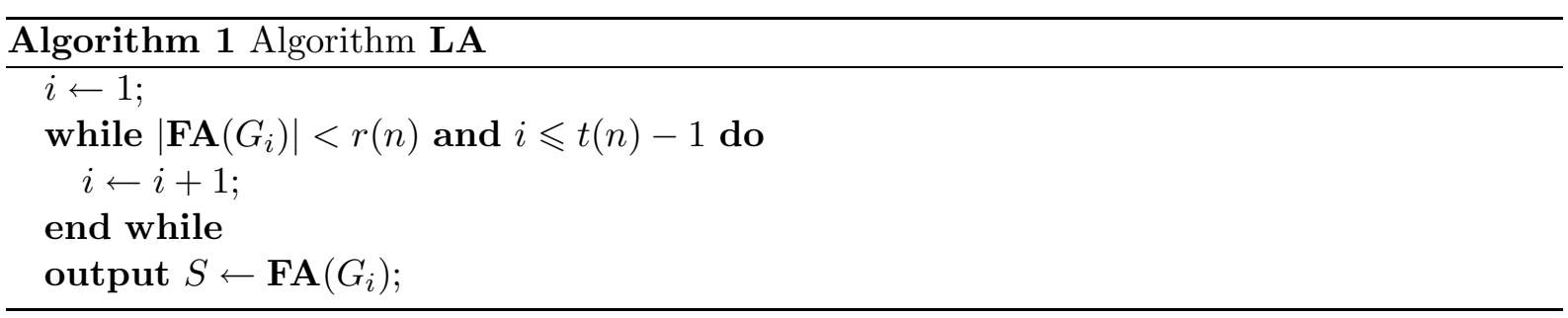

If $\exists i,\left|\mathbf{F A}\left(G_{i}\right)\right| \geqslant r(n)$, then

$$
c_{\mathbf{L A}}(G) \geqslant \frac{|S|}{\beta(G)} \geqslant \frac{r(n)}{n}
$$

On the other hand, if, $\forall i \leqslant t(n),\left|\mathbf{F A}\left(G_{i}\right)\right|<r(n)$, then

$$
\beta\left(G_{i}\right) \leqslant \frac{\left|\mathbf{F A}\left(G_{i}\right)\right|}{\rho\left(n_{i}\right)} \leqslant \frac{r(n)}{\rho(n, \Delta(G))}
$$

Since we deal with hereditary properties, the restriction of an optimal solution on $G$ in every $G_{i}$, forms a feasible solution for $G_{i}$; consequently, by expression (2) we get

$$
\beta(G) \leqslant \sum_{i=1}^{t(n)} \beta\left(G_{i}\right) \leqslant \frac{t(n) r(n)}{\rho(n, \Delta(G))}
$$

Since $\left|\mathbf{F A}\left(G_{t(n)}\right)\right| \geqslant 1$, we have $|S| \geqslant 1$ and expression (3) gives

$$
\frac{|S|}{\beta(G)} \geqslant \frac{\rho(n, \Delta(G))}{t(n) r(n)}
$$

Combining expressions (1) and (4), we get

$$
c_{\mathbf{L A}}(G) \geqslant \min \left\{\frac{r(n)}{n}, \frac{\rho(n, \Delta(G))}{t(n) r(n)}\right\}
$$

\footnotetext{
${ }^{2}$ The approximation ratio of a polynomial time approximation algorithm $\mathbf{A}$ for an instance $I$ of an NPO problem is the ratio of the value of the solution produced by $\mathbf{A}$ on $I$ to the value of the optimal solution of $I$.
} 
Setting

$$
r(n)=\sqrt{\frac{n \rho(n, \Delta(G))}{t(n)}}
$$

one obtains the result claimed.

Starting from theorem 1 the following results can be deduced.

Corollary 1. When $t(n)$ is a fixed constant, then

$$
c_{\mathbf{L} \mathbf{A}}(G) \geqslant O\left(\sqrt{\frac{\rho(n, \Delta(G))}{n}}\right)
$$

- if $\rho(n)=O(\log n / n)([3])$, then

$$
c_{\mathbf{L A}}(G) \geqslant O\left(\sqrt{\frac{\log n}{n}}\right) .
$$

In the case where $\rho$ depends only in $n$ and is convex one can improve the result of theorem 1 .

Theorem 2. When $\rho(n)$ is convex, then

$$
c_{\mathbf{L A}}(G) \geqslant O\left(\sqrt{\frac{\rho\left(\frac{n}{t(n)}\right)}{n t(n)}}\right) .
$$

Note that the discussion above can be interpreted in terms of reduction of LHG to HG, transforming any approximation ratio $\rho(n, \Delta(G))$ for HG to a competitivity ratio $(\rho(n, \Delta(G)) /(n t(n)))^{1 / 2}$ for LHG.

\section{On-line independent set}

\subsection{Vertices arrive one-by-one}

We now suppose that vertices arrive one-by-one together with the edges linking them to the vertices already revealed. Once a vertex arrived, an on-line algorithm has to definitely decide if it will include it in the solution under construction. The simplest such algorithm is algorithm 2 where we suppose that vertices are indexed in the order of their arrival.

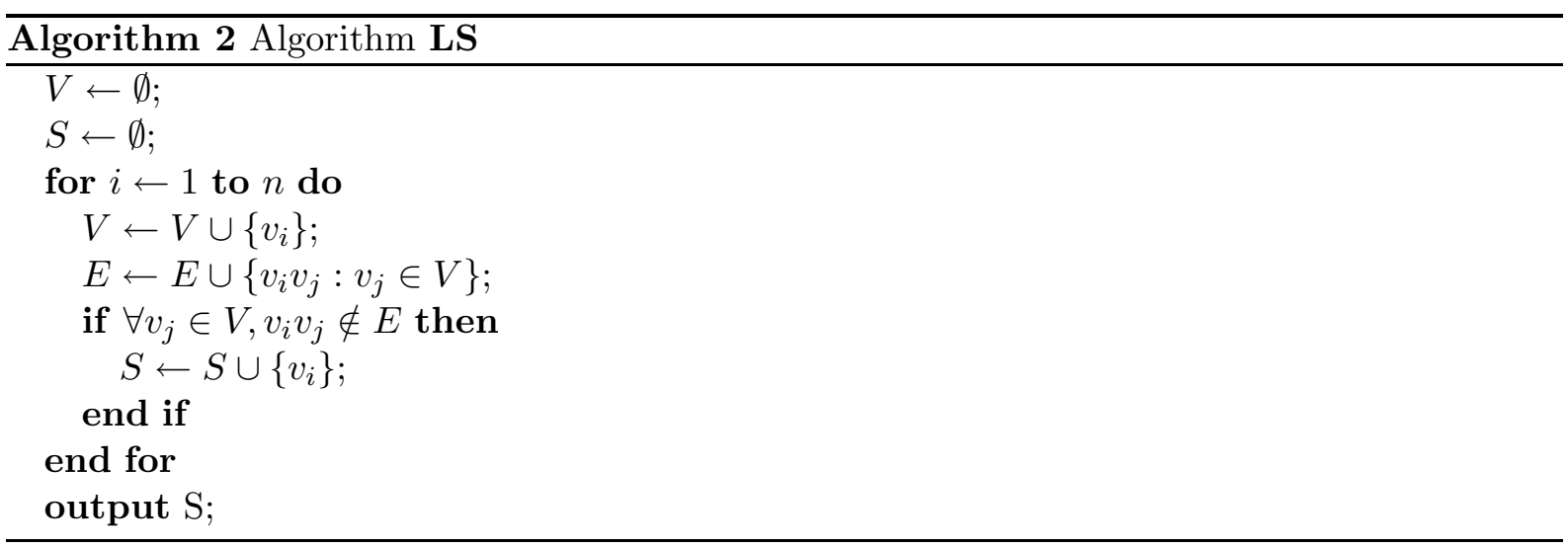

In what follows, dealing with IS, we denote by $\alpha(G)$ the independence number of $G$. It is easy to see that the set $S$ computed by algorithm $\mathbf{L S}$ is a maximal independent set. Moreover, it is well-known that maximal independent sets are of size at least $\alpha(G) / \Delta(G)$. 
Proposition 1. $c_{\mathbf{L S}} \geqslant 1 / \Delta(G)$.

Theorem 3. Under the hypothesis that vertices are revealed one-by-one, then, $\forall \epsilon>0$, no on-line algorithm can guarantee competitivity ratio greater than $1 /(\Delta(G)-\epsilon)$.

Proof. (Sketch.) Choose some $\epsilon>0$ and set $L=\lceil 1 / \epsilon\rceil$. Consider $\Delta \in \mathbb{N}$ and set $n=L(\Delta+1)$. It could happen that:

- whenever a set $S$ of $\Delta$ independent vertices has been revealed but has not been taken in the solution under construction, then the next vertex revealed is the root of a tree of depth 1 having the vertices of $S$ as leaves;

- whenever a vertex $v$ is included in the solution and the number of vertices already revealed is less than $n-\Delta$, then the $\Delta$ next revealed vertices together with $v$ form a tree of depth 1 rooted at $v$;

- suppose that less than, or equal to, $\Delta+1$ vertices, $v_{0}, v_{1}, \ldots, v_{\Delta}$ remain to be revealed in this order, and that $v_{0}, \ldots, v_{i}, 1 \leqslant i \leqslant \Delta-1$, form an independent set and $v_{0}, \ldots, v_{i-1}$ have not been included in the solution under construction, whereas $v_{i}$ is included; then, the subgraph of $G$ induced by $v_{0}, v_{1}, \ldots, v_{\Delta}$ can be a tree $T$ of depth 2 such that:

$-v_{i}$ is the root of $T$,

- the first level of $T$ consists of $v_{i+1}, \ldots, v_{\Delta}$ where $v_{i+1}$ is internal and $v_{i+2}, \ldots, v_{\Delta}$ are leaves;

- the second level of $T$ consists of leaves $v_{0}, \ldots, v_{i-1} ;$.

- the final graph $G$ has no edge other than the ones of the items above.

Remark that the graph so-constructed is of order $n$ and has maximum degree $\Delta(G)=\Delta$.

It is easy to see that $\alpha(G) \geqslant L \Delta(G)-1$ and the solution constructed contains no more than $L$ vertices. Then, the competitivity ratio is the one claimed.

\subsection{Vertices are revealed by clusters}

As we have already mentioned, IS is one of the most known instantiations of HSG. Consequently, the result of theorem 1 applies here. The best approximation ratio (in terms of $n$ ) is guaranteed by the algorithm of [1] and is of $O\left(\log ^{2} n / n\right)$. Using this ratio in theorem 1 and corollary 1 (in other words, considering the algorithm of [1] in place of FA in algorithm LA), the following results hold.

\section{Theorem 4.}

1 .

$$
c_{\mathbf{L A}}(G) \geqslant O\left(\frac{\log n}{n \sqrt{t(n)}}\right)
$$

2. when $t(n)$ is a fixed constant

$$
c_{\mathbf{L A}}(G) \geqslant O\left(\frac{\log n}{n}\right)
$$

3. since $\log ^{2} n / n$ is convex (for $n \geqslant 31$ ), if $t(n) \leqslant O(n / \log n)$, then,

$$
c_{\mathbf{L A}}(G) \geqslant O\left(\frac{\log \log n}{n}\right) \text {. }
$$




\section{On-line maximum clique}

In what follows, we denote by $\kappa(G)$ the size of a maximum clique of $G$ and, for $V^{\prime} \subseteq V$, we denote by $G\left[V^{\prime}\right]$, the subgraph of $G$ induced by $V^{\prime}$.

\subsection{Vertices arrive one-by-one}

We suppose here that the final graph does not contain isolated vertices. Let us consider algorithm 3, where, as in section 3.1, we suppose that vertices are indexed in the order of their arrival. Since the final graph $G$ has no isolated vertices, $|K| \geqslant 2$. On the other hand, $\kappa(G) \leqslant \Delta(G)+1$.

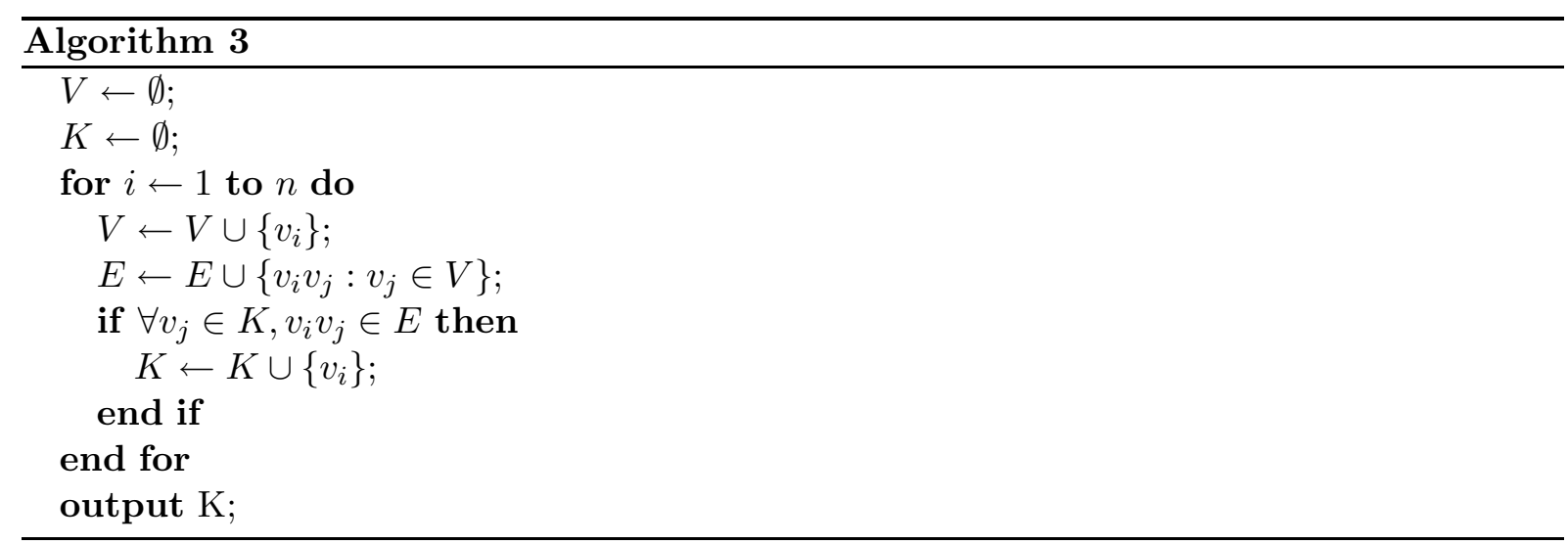

Consequently,

$$
c_{\mathbf{L C l}}(G) \geqslant \frac{2}{\Delta(G)+1}
$$

\subsection{Vertices arrive by clusters}

Of course, $\mathrm{Cl}$ being another well-known instantiation of HSG, the results of section 3.2 apply identically here. In what follows, we will use polynomial reductions in order to obtain competitivity ratios functions of $\Delta(G)$ for LCl.

\subsubsection{Reducing on-line clique to (off-line) clique}

Applying theorem 1 and taking into account that $\kappa(G) \leqslant \Delta(G)+1$, we get the following theorem.

Theorem 5. Under the on-line model where vertices arrive by clusters, IS reduces to LCl. This reduction transforms every approximation ratio $\rho(n)$ for IS to a competitivity ratio

$$
O\left(\sqrt{\frac{\rho(\Delta(G))}{t(n) \Delta(G)}}\right)
$$

for $\mathrm{LCl}$.

In [2], we devise a polynomial time approximation algorithm guaranteeing approximation ratio $O\left(\log ^{2} \Delta(G) / \Delta(G)\right)$ for Cl. Using this results together with theorem 5 we get the following corollary.

Corollary 2. When $t(n)$ is a fixed constant, the competitivity ratio

$$
O\left(\frac{\log \Delta(G)}{\Delta(G)}\right)
$$

can be guaranteed for $\mathrm{LCl}$. 


\subsubsection{Reducing on-line independent set to on-line clique}

Revisit now algorithm LA, instantiate algorithm FA by the algorithm of [1] and consider that, instead of $G_{i}$, the input of $\mathbf{L} \mathbf{A}$ is $\bar{G}_{i}$ (since $G_{i}$ is known, so does $\bar{G}_{i}$ ). Then, using the complementarity relation independent set in $G_{i}$ - clique in $\bar{G}_{i}$, one can easily deduce the following theorem 6.

Theorem 6. There exist a reductions from LCl to LIS (and vice-versa) preserving competitivity ratios, functions of $n$.

An immediate consequence of theorem 6 is that the results of theorem 4 holds also for LCl.

We now show how a competitivity ratio $c(n)$ for LIS can be transformed into a competitivity ratio $c^{\prime}(\Delta(G))$ for $\mathrm{LCl}$. Note that $n$ is the cardinality of the final graph.

We shall use the following notations. We set $[V]_{i}=V_{1}, \ldots, V_{i}$ and denote by $\left(G,[V]_{t(n)}\right)$, an instance of LIS and by $[G]_{i}$, the graph $G\left[V_{1} \cup \ldots \cup V_{i}\right]$. Given an on-line IS-algorithm LA, an integer $i$, a graph $H$ of order $|H|$, the vertices of which are partitioned into $i$ non-empty sets $V_{1}, \ldots, V_{i}$, and given $n \in \mathbb{N}$ such that $i \leqslant t(n)$ and $|H| \leqslant n$, we denote by $\left\{\mathbf{L A}_{i}\left(H,[V]_{i}, n\right)\right\}$ and by $\left\{[\mathbf{L A}]_{i}\left(H,[V]_{i}, n\right)\right\}$, the set of vertices introduced in the solution computed by $\mathbf{L} \mathbf{A}$ during step $i$ and during steps $1, \ldots, i$, respectively, when the $i$ first vertex-clusters revealed are $V_{1}, \ldots, V_{i}$ and the finally revealed graph is of order $n$. For every instance $\left(G,[V]_{t(n)}\right)$ of LIS and for every vertex $v \in V$, we denote by $r(v)$ the number such that $v \in V_{r(v)}$. We set $N_{v}=\{(u \in\{v\} \cup \Gamma(v)): r(u) \geqslant r(v)\}$, where $\Gamma(u)$ denotes the set of neighbors of $u$ in $G$. By $[\Gamma(v)]_{i}$, we denote the set of neighbors of $v$ in $[G]_{i}$.

Lemma 1. Let $\boldsymbol{L} \boldsymbol{A}$ be an on-line algorithm for LIS guaranteeing competitivity ratio $c(n)$ when running on a graph of order $n$ (only $n$ is known at the beginning of $\boldsymbol{L} \boldsymbol{A}$ ). Let $i \leqslant t(n)$ such that $\left|\cup_{j=1}^{i} V_{j}\right| \leqslant n / 2$. Then:

1. if $\left\{[\mathbf{L A}]_{i}\left([G]_{i},[V]_{i}, n\right)\right\}=\emptyset$, then

$$
\frac{1}{\alpha\left([G]_{i}\right)} \geqslant c(n)
$$

2. if $\left\{[\mathbf{L A}]_{i}\left([G]_{i},[V]_{i}, n\right)\right\} \neq \emptyset$, then

$$
\frac{1}{\frac{n}{2}} \geqslant c(n)
$$

Theorem 7. There exist a reduction from LCl to LIS transforming competitivity ratios $c(n)$ into competitivity ratios $c(\Delta(G)+1) / 2$.

Proof. (Sketch.) Suppose an algorithm LISA achieving competitivity ratio $c(n)$ when running on an instance $\left(G,[V]_{t(n)}\right)$ of LIS, and consider algorithm LClA (algorithm 4) computing a clique in $G$.

Set $W_{v}=(\{v\} \cup \Gamma(v)) \cap N_{v}, v \in V$. Also, observe that, if the output-clique $K$ is computed in line 25 during the $i$ th execution of the repeat-loop of line 5 , then it contains only vertices of $V_{i}$. Clearly, if the if-condition of line 24 is true, then bool1 becomes true and the algorithm will finish at the end of this iteration. But if the clique computed contains at least one vertex of $V_{j}$ for $j<i$, then during the $j$ th iteration, a non-empty clique would have been computed and the algorithm would have finished without ulterior iterations of the repeat-loop.

We distinguish two cases whether bool1 is true or not at line 34 of algorithm LClA. 


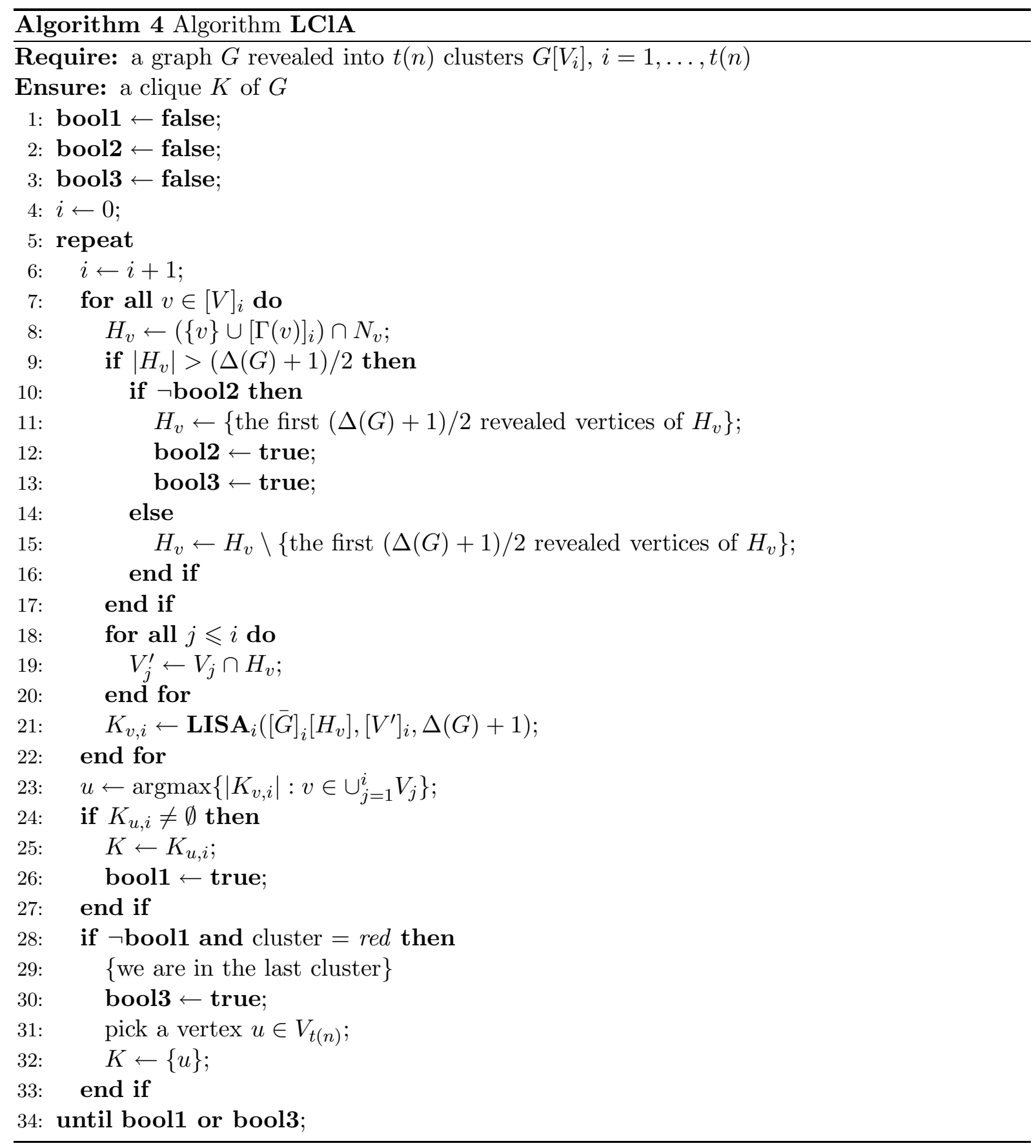




\subsubsection{Variable bool1 is true}

Then, the clique computed by algorithm LClA has been produced in line 25. Consider $u$ and $i$ such that

$$
K_{u, i}=K=\left\{\mathbf{L I S A}_{i}\left([\bar{G}]_{i}\left[H_{v}\right],\left[V^{\prime}\right]_{i}, \Delta(G)+1\right)\right\}
$$

while, for $j<i$,

$$
\left\{\operatorname{LISA}_{j}\left([\bar{G}]_{j}\left[H_{v}\right],\left[V^{\prime}\right]_{j}, \Delta(G)+1\right)\right\}=\emptyset .
$$

Expressions (6) and (7) mean

$$
\left\{\operatorname{LISA}_{i}\left([\bar{G}]_{i}\left[H_{v}\right],\left[V^{\prime}\right]_{i}, \Delta(G)+1\right)\right\}=\left\{[\mathbf{L I S A}]_{i}\left([\bar{G}]_{i}\left[H_{v}\right],\left[V^{\prime}\right]_{i}, \Delta(G)+1\right)\right\} .
$$

Moreover, remark that

$$
\left|[\bar{G}]_{i}\left[H_{v}\right]\right| \leqslant \frac{\Delta(G)+1}{2} .
$$

Then, using item 2 of lemma 1, we have

$$
|K| \geqslant c(\Delta(G)+1) \frac{\Delta(G)+1}{2} \geqslant c(\Delta(G)+1) \frac{\left|K^{*}\right|}{2}
$$

where $K^{*}$ denotes a maximum-order clique of $G$.

\subsubsection{Variable bool1 is false}

Then $K$ contains only one vertex. Let $x \in \operatorname{argmin}\left\{r(y): y \in K^{*}\right\}$. We partition set $W_{x}$ into two sets $W_{x}^{1}$ and $W_{x}^{2}$, set $W_{x}^{1}$ containing the first $(\Delta(G)+1) / 2$ revealed vertices of $W_{x}$ (if $\left|W_{x}\right|<(\Delta(G)+1) / 2$, then $\left.W_{x}^{1}=W_{x}, W_{x}^{2}=\emptyset\right)$. Since $\left|W_{x}\right|<\Delta(G)+1$ and $K^{*} \subseteq W_{x}$, at most one between $W_{x}^{1}$ and $W_{x}^{2}$, includes the half of $K^{*}$; let us denote by $U_{x}$ this set. Remark also that during one of the executions of the repeat-loop, $H_{x}=U_{x}$. Since $\forall v \in V$ and $\forall i \leqslant t(n)$,

$$
\left\{\operatorname{LISA}_{i}\left([\bar{G}]_{i}\left[H_{v}\right],\left[V^{\prime}\right]_{i}, \Delta(G)+1\right)\right\}=\emptyset
$$

(a fortiori this remains true also for $x$ ), one vertex is sufficient in order that algorithm LISA guarantees the competitivity ratio when called on a graph the final order of which is at most $\Delta(G)+1$. Consequently, from item 1 of lemma 1:

$$
\frac{1}{\frac{\left|K^{*}\right|}{2}} \geqslant c(\Delta(G)+1) \Longleftarrow \frac{|K|}{\left|K^{*}\right|} \geqslant \frac{c(\Delta(G)+1)}{2}
$$

So, in both cases, on the hypothesis that there exists an on-line algorithm achieving competitivity ratio $c(n)$, the solution computed by algorithm LClA guarantees competitivity ratio $c(\Delta(G)+$ 1) $/ 2$.

\section{References}

[1] B. B. Boppana and M. M. Halldórsson. Approximating maximum independent sets by excluding subgraphs. BIT, 32(2):180-196, 1992.

[2] M. Demange and V. T. Paschos. Maximum-weight independent set is as "well-approximated" as the unweighted one. Technical Report 163, LAMSADE, Universit Paris-Dauphine, 1999.

[3] M. M. Halldórsson. Approximations via partitioning. JAIST Research Report IS-RR-950003F, Japan Advanced Institute of Science and Technology, Japan, 1995. 